\title{
Relación entre obesidad, adipocitoquinas y osteoatrosis: Una revisión
}

\author{
Relationship between obesity, adipocykines and osteoarthritis: Review
}

Yoccner Edilson Quenan ${ }^{1,2^{*}}$ orcid.org/0000-0002-8047-0306

José Henry Osorio ${ }^{1,2}$ orcid.org/0000-0002-6875-3215

1. Laboratorio de Investigación en Metabolismo, Universidad de Manizales. Maestría en Ciencias Biomédicas, Universidad de Caldas. Manizales, Colombia

2. Laboratorio de Investigación Bioquímica Clínica, Universidad de Caldas. Manizales, Colombia

\section{Resumen}

Introducción: Algunas adipocitoquinas expresadas por osteoblastos, condrocitos y sinoviocitos son responsables de la inflamación y degradación de la matriz extracelular en las articulaciones, generando osteoartrosis. Objetivo: Revisar el estado actual del conocimiento en torno a la relación que existe entre obesidad, adipocitoquinas y osteoartrosis. Materiales y métodos: Se realizó una búsqueda electrónica en las bases de datos de BBCS-LILACS, PubMed, IB-PsycINFO, IB-SSCI, IB-SciELO, Scopus y Science Direct en inglés y español, sin limitar la búsqueda por fecha. Se incluyeron ensayos clínicos, metaanálisis, revisiones, reportes de casos y artículos clásicos, relacionados con la obesidad y sus implicaciones, osteoartrosis, adipocitoquinas, tejido adiposo e inflamación articular. Resultados: Se encontró 822 artículos, 78 documentos cumplieron los criterios de selección por la pertinencia de la información. El presente trabajo se dividió en tres secciones a saber: osteoartrosis y obesidad, osteoartrosis e inflamación y osteoartrosis y adipocitoquinas. Conclusión: En la actualidad, la obesidad está asociada con el desarrollo de la osteoartrosis, ya que se desencadena una serie de procesos inflamatorios mediados por las adipocitoquinas. Varios estudios recomiendan el desarrollo de investigaciones clínicas para entender el comportamiento de las adipocitoquinas antes y durante el desarrollo de la osteoartrosis.

Palabras clave: Obesidad; inflamación; metabolismo; tejido adiposo; osteoartrosis. (Fuente: DeCS, Bireme).

\begin{abstract}
Introduction: Some adipocytokines expressed by osteoblasts, chondrocytes and synoviocytes are responsible of the inflammation and degradation of the extracellular matrix in the joints, which generates osteoarthritis. Objective: To review the current state of knowledge around the relationship between obesity, adipocytokines and osteoarthritis. Materials and methods: An electronic search was carried out in the databases of BBCS-LILACS, PubMed, IBPsycINFO, IB-SSCI, IB-SciELO, Scopus and Science Direct in English and Spanish, without limiting the search by date. Clinical trials, meta-analyses, reviews, case reports and classic articles related to obesity and its implications were included as well as osteoarthritis, adipocytokines, adipose tissue and joint inflammation. Results: 822 articles were found; however, 78 documents met the selection criteria for the relevance of the information. This work was divided into three sections: Osteoarthritis and obesity, osteoarthritis and inflammation and osteoarthritis and adipocytokines. Conclusion: Nowadays, obesity is associated with the development of osteoarthritis, as it triggers a series of inflammatory processes mediated by the adipocytokines. Several studies recommend the development of clinical research to understand the behavior of adipocytokines before and during the development of osteoarthritis.
\end{abstract}

Keywords: obesity; inflammation; metabolism; adipose tissue; osteoarthritis. (Source: DeCS, Bireme). 


\section{Introducción}

La osteoartrosis (OA), también conocida como artrosis $\mathrm{u}$ osteoartritis, es una enfermedad articular caracterizada por dolor crónico degenerativo en una o más articulaciones, por lo que el órgano blanco es el cartílago, aunque pueden estar comprometidas todas las estructuras que hacen parte de la articulación(1). La OA es la más común de las enfermedades reumáticas crónicas, se presenta un desgaste articular del cartílago producto de varias causas(2): congénitas, adquiridas, inmunológicas, metabólicas, inducidas entre otras; ese desgaste se manifiesta en dolor, deformidad y limitación funcional especialmente en las articulaciones que tienen mayor movilidad o soportan mayor peso del normal(3).

La OA es una patología articular degenerativa con deterioro gradual y progresivo del cartílago, con reacción proliferativa del hueso subcondral e inflamación de la membrana sinovial, así como daño en meniscos, tendones, músculos y nervios asociados con la articulación afectada( ${ }^{(4)}$. En la fase temprana de la enfermedad los eventos patológicos son dinámicos, presentándose transformaciones estructurales que previenen la inestabilidad de la articulación, pero a medida que avanza la enfermedad, las estructuras afectadas experimentan cambios patológicos irreversibles que llevan finalmente al deterioro funcional total de la articulación. La artrosis afecta a todas las estructuras de la articulación, se presenta la pérdida del cartílago hialino articular, hay remodelación del hueso subcondral, junto a un estiramiento capsular y debilitamiento de los músculos periarticulares $(5,6)$; estos desencadenan cambios homeostáticos y se asocian con el aumento en la producción de factores inflamatorios y trastornos del sistema endocrino.

Estudios recientes ponen de manifiesto la relación entre los niveles de adipocitoquinas y las enfermedades osteoarticulares como la $\mathrm{OA}^{(7,8)}$. Entre las adipocitoquinas se encuentra la adiponectina, resistina, leptina y visfatina, estas se producen generalmente en el tejido adiposo blanco, y también son expresadas por osteoblastos, condrocitos y sinoviocitos y se han descrito como biomoléculas que inciden en la inflamación de las articulaciones y degradación de la matriz extracelular(9). Los niveles de estas adipocitoquinas encontrados en el líquido sinovial se correlacionan con la evolución y severidad de la $\mathrm{OA}^{(10)}$.

En la actualidad los estudios centran su atención en la inflamación articular y su relación en la patogénesis de la $\mathrm{OA}$, además se involucra a todos los tejidos articulares incluyendo cartílago, hueso, meniscos, sinovia, estructuras capsulares, tendones y ligamentos(11). A parte de la lesión articular, también se estudia la relación de la obesidad y la edad como factores de riesgo de la $\mathrm{OA}^{(12)}$, de tal manera que los cambios metabólicos debidos al tejido graso, pueden contribuir al desarrollo de la enfermedad inducida por la obesidad(13). Existen reportes donde se relaciona la obesidad con $\mathrm{OA}$ en articulaciones que no soportan peso, por ejemplo, la de la mano, hombro, evidenciando que los factores metabólicos también contribuyen a la alta prevalencia de $\mathrm{OA}$ en humanos obesos(14).

El objetivo de este trabajo es revisar el estado actual del conocimiento en torno a la relación que existe entre obesidad, adipocitoquinas y osteoartrosis, teniendo como referencia las alteraciones metabólicas generadas por la obesidad, relacionadas con una respuesta inflamatoria crónica especialmente en el líquido sinovial.

\section{Materiales y métodos}

Estudio observacional, retrospectivo, transversal y analítico. Se realizó una búsqueda sistemática en las bases de datos de BBCS-LILACS, PubMed, IB-PsycINFO, IB-SSCI, IB-SciELO, Scopus y Science Direct en inglés y español, sin limitar la búsqueda por fecha. La búsqueda bibliográfica inicio desde junio de 2015 hasta julio de 2016. Se incluyeron ensayos clínicos, metaanálisis, revisiones, reportes de casos, artículos clásicos y textos de libros, artículos publicados sobre obesidad y sus implicaciones, osteoartrosis, adipocitoquinas, tejido adiposo e inflamación 
articular. Se encontró un total de 822 artículos que se organizaron en una matriz en Excel, la cual contenía los ítems: número de identificación, nombre del artículo, autor (es), año publicación, revista (Tabla 1); después se seleccionaron los que trataban sobre los criterios centrales de la revisión: obesidad, adipocitoquinas y osteoartrosis, lo anterior teniendo en cuenta las recomendaciones PRISMA; después se organizó otra matriz con los documentos seleccionados y se adicionó el ítem resumen de cada artículo, para posterior lectura, resumen y análisis de la información de interés.

Tabla 1. Proceso de selección de artículos

\begin{tabular}{|c|c|c|c|c|c|c|c|}
\hline BBCS-LILACS & PubMed & IB-PsycINFO & IB-SSCI & IB-SciELO & Scopus & Science Direct & Total \\
\hline 87 & 175 & 126 & 78 & 102 & 76 & 178 & 822 \\
\hline Duplicados & & & & & & & 32 \\
\hline \multicolumn{2}{|c|}{ Eliminados por titulo } & & & & & & 420 \\
\hline \multicolumn{2}{|c|}{ Sin texto completo disponible } & & & & & & 291 \\
\hline \multicolumn{2}{|c|}{ Artículos seleccionados } & & & & & & 78 \\
\hline \multicolumn{2}{|c|}{ Reportes de caso } & Revisiones & & Editoriales & & Originales puk & dos \\
\hline & 1 & & 7 & & 3 & & 67 \\
\hline
\end{tabular}

\section{Resultados y discusión}

Los documentos que cumplieron con los criterios de selección osteoartrosis y obesidad osteoartrosis e inflamación y osteoartrosis y adipocitoquinas fueron 78, por lo cual los resultados se dividieron en tres secciones a saber:

\section{Osteoartrosis y obesidad}

Una de las consecuencias de la obesidad es el aumento considerable de la carga mecánica sobre las articulaciones que soportan la mayoría de peso. Estudios sugieren una relación directa entre pacientes obesos y $\mathrm{OA}$ de rodilla $\mathrm{y}$ cadera(15), el riesgo de padecer $\mathrm{OA}$ de rodillas aumenta cuando el índice de masa corporal (IMC) es $\geq 30 \mathrm{~kg} / \mathrm{m}^{2(16)}$, es decir que los pacientes que presentan valores iguales 0 superiores tienen 4,2 veces más posibilidades de tener $\mathrm{OA}$ de rodillas en comparación con las personas con un IMC normal. También se observa que el IMC elevado se asocia con una rápida progresión de la $\mathrm{OA}^{(17)}$; la $\mathrm{OA}$ que se presenta en las articulaciones de la mano no se debe en gran medida a la sobrecarga sino a factores inflamatorios y metabólicos(18).

Como se ha mencionado anteriormente, el aumento en la carga biomecánica que resisten las articulaciones, producto del incremento de la masa grasa se asocia con la aparición de la $\mathrm{OA} \mathrm{y}$ potencia el desarrollo de comorbilidades cardiovasculares(19), esa carga anormal que soporta directamente el cartílago se relaciona con desequilibrios inflamatorios y metabólicos, esto a partir de la activación de las citoquinas pro inflamatorias como mecanismo de defensa(20). En este proceso, los condrocitos los cuales son un tipo de célula que se encuentran en el cartílago, encargados de mantener la matriz cartilaginosa, a través de la producción de sus principales compuestos: colágeno y proteoglicanos(21), responden a esa carga anormal a través de sus mecanoreceptores, produciendo la síntesis de citocinas inflamatorias, las cuales contribuyen a la degradación del cartílago articular(20). Una de las consecuencias de la obesidad, es aumentar las cargas que soportan las articulaciones y por ende se desencadenan procesos inflamatorios en respuesta a esa sobrecarga; además la obesidad está relacionada con estados inflamatorios y oxidativos los cuales alteran las concentraciones de las diversas biomoléculas(22). Entre las biomoléculas que se modifican encontramos a las adipocitoquinas las cuales se sintetizan en el tejido adiposo(23), este último un órgano metabólicamente activo, posee una marcada actividad endocrina, paracrina y autocrina(24). La 
unidad funcional del tejido adiposo son los adipocitos, los cuales tienen la capacidad de secretar adipocitocinas, y sustancias vasoactivas. En las primeras encontramos a la adiponectina, resistina, visfatina y leptina, estas se correlacionan con el peso (se mide a través del IMC), la circunferencia de la cintura, la trigliceridemia, la insulinemia y la resistencia a la insulina; además, estas citocinas se encuentran implicadas en la regulación del peso corporal (leptina, adiponectina), la activación del sistema inmune, Factor de Necrosis Tumoral (TNF), interleuquinas 1 y 6 (IL-1 IL-6), resistina (ADSF por sus siglas en inglés: Adipose Tissue Specific Secretory Factor) y la función vascular (angiotensina, PAI-1)(25).

El tejido adiposo blanco en adelante $\mathrm{TAB}$, está relacionado con la secreción de más de 50 factores con actividad similar a la de citoquinas, las cuales están involucradas en varios procesos fisiológicos y patológicos, incluidas la inmunidad y la inflamación(26), por ejemplo, producto del sobrepeso, se ejerce una presión mecánica sobre las articulaciones en especial sobre el hueso subcondral estimulando la expresión de genes que codifican para IL-6, IL-8, Ciclooxigenasa-2 (COX-2), factor de crecimiento del fibroblasto-2 (FGF-2), metaloproteinasas de matriz (MMP) como MMP-3, MMP-9 y MMP-13 y ligando del Receptor Activador del Factor Nuclear $\kappa \mathrm{B}$ (RANKL), además reduce la expresión de la osteoprotegerina (OPG) ${ }^{(27)}$.

Durante el curso de la OA se presentan cambios que incrementan el consumo de energía, reacomodan el tejido graso, aumentan la gluconeogénesis, y catabolismo proteico entre otros, que aceleran la morbimortalidad de los pacientes(18).

\section{Osteoartrosis e inflamación}

El sobrepeso y los procesos metabólicos producto de la obesidad en las articulaciones, ocasionan lesión en el cartílago por debilitamiento de los proteoglicanos y daño en la red de colágeno(28), desencadenando una serie de procesos catabólicos que disminuyen la síntesis de proteínas de la matriz del cartílago e incrementan aún más la expresión de mediadores proinflamatorios y por ende la destrucción progresiva del cartílago articular(29). Seguidamente se genera la activación de unas señales intracelulares mediante activación de unas interleuquinas, las cuales estimulan la liberación de moléculas de oxígeno reactivo, óxido nítrico encargados de la destrucción de los condrocitos; una vez se presente esta etapa, se activan moléculas que controlan a la MMP-13, la

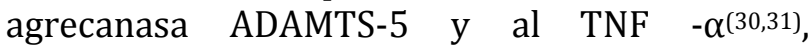
recientemente se han identificado otra agrecanasa, la ADAMTS-7, que destruye las proteínas oligoméricas de la matriz del cartílago (COMP) in vivo. Estos fenómenos potencian las funciones catabólicas del TNF- $\alpha$ y MMPs, por lo que se desencadena la $\mathrm{OA}$ la cual tiene la característica de ser progresiva y degenerativa(32, 33).

La inflamación en la OA, es un indicio del daño del cartílago articular y por ende de la progresión de la enfermedad, se presentan unos signos y síntomas clásicos: dolor de las articulaciones, inflamación y rigidez, que también son indicadores de la sinovitis(34).

En la sinovitis, la membrana sinovial previa infiltración de células mononucleares, libera mediadores proinflamatorios, incluyendo la IL$1 \beta$, TNF- $\alpha$, IL- 6 y quimiocinas, estos contribuyen a la progresión de la OA, sin embargo, esa liberación es común en la fase inicial y tardía de la enfermedad(35). La actividad de esos factores solubles altera los procesos metabólicos que se producen en el cartílago, por lo tanto incrementa el catabolismo de los tejidos articulares, que conlleva a un mayor daño articular(36). Durante el desarrollo de la OA, existe un aumento de las células endoteliales, que estimula la formación de nuevos vasos sanguíneos, existe una estrecha relación entre la angiogénesis y los procesos inflamatorios, ya que estos últimos demandan del transporte de células inflamatorias, de nutrientes y de oxígeno, de esa manera se crea una dependencia entre la actividad inflamatoria y la angiogénesis(37), además esta última contribuye a que la inflamación pase de un estado agudo a un crónico(38). 


\section{Osteoartrosis y adipocitoquinas}

La OA y síndrome metabólico están relacionados, de allí que factores metabólicos sistémicos asociados con la obesidad contribuyen a la respuesta inflamatoria y por ende a la patogénesis de la enfermedad(39), dentro de este proceso como se mencionó anteriormente, el TAB juega un papel importante, ya que no sólo actúa como tejido de almacén, sino que secreta varias adipocitoquinas las cuales tienen incidencia sobre la $\mathrm{OA}^{(40)}$. Debido a ese rol metabólico que ejerce el $\mathrm{TAB}$, las principales adipocinas involucradas en OA son: leptina, adiponectina, resistina, visfatina, lipocalina-2, vaspina, apelina, omentina, quemerina $y$ amiloide sérico $\mathrm{A} 3$, siendo las más estudiadas: la leptina, adiponectina, visfatina y resistina(41).

Estas adipocinas proinflamatorias están relacionadas con el desarrollo de la inflamación en las personas obesas, generando varios fenómenos metabólicos que incluyen complicaciones cardiovasculares y enfermedades inflamatorias autoinmunes. A continuación, se describe la incidencia de las adipocinas en la respuesta inflamatoria e inmune, centrando la atención en la OA.

La leptina, es una hormona peptídica no glicosilada de $16 \mathrm{kDa}$, secretada principalmente por la placenta o tejido adiposo blanco(42), es codificada por el gen obeso, pertenece a la familia de las citocinas y su concentración sérica está relacionada directamente con la cantidad de grasa corporal, es decir que, un aumento en el tejido adiposo eleva la producción de leptina(26). En humanos y animales esta actúa uniéndose a sus receptores específicos (Ob-RS) los cuales se encuentran a nivel del cerebro y en tejidos periféricos(43), se encarga de la regulación del peso corporal mediante la inhibición de ingesta de alimento, debido a la supresión del apetito y el aumento del gasto de energía, a través de la estimulación de centros hipotalámicos y la disminución de la secreción de neuropéptido $Y$ (NPY)(44). En la OA, la leptina tiene relación en especial con el metabolismo del cartílago(45), en investigaciones clínicas se ha encontrado mayor concentración de esta hormona en la grasa infrapatelar y el tejido sinovial de personas con
OA en comparación con los controles de individuos sanos(46). En el cartílago de pacientes con OA los condrocitos producen mayor cantidad de leptina en comparación con el cartílago normal ${ }^{(47,48)}$, de tal manera que a medida que avanza la enfermedad se genera mayor destrucción del cartílago y por ende los niveles de la hormona son más altos en etapas avanzadas de la $\mathrm{OA}^{(49)}$, este proceso proinflamatorio lo realiza estimulando unas moléculas de adhesión celular VCAM-1, así que permite la adhesión de leucocitos e infiltración de monocitos a las articulaciones afectadas(50), además, la leptina promueve la secreción de IL-8 en los condrocitos que contribuyen a la formación del gradiente quimiotáctico presente en las articulaciones inflamadas(51). El incremento de los niveles de leptina provocan aumento en las concentraciones de MMP-9, MMP-14 y óxido nítrico, estos afectan el metabolismo de los condrocitos y por ende fomentan la $\mathrm{OA}^{(52)}$. Varios estudios reportan una estrecha relación entre la leptina y la OA, esta podría ser un biomarcador conveniente para predecir la gravedad de la enfermedad(53). La OA, es más predominante en las mujeres, puesto que ellas tienen mayores concentraciones de leptina que los hombres(54).

La resistina, es una proteína de $12,5 \mathrm{kDa}$, también se conoce como ADSF o FIZZ-3 porque se encuentra en la zona inflamatoria $3^{(55)}$; es una proteína rica en cisteína, compuesta por 108 aminoácidos que circulan como una proteína dimérica(56); la producción de resistina se debe en gran parte a los macrófagos, mientras que en los animales predomina la producción en el $\mathrm{TAB}(57,58) ;$ la resistina se produce a partir de las células proinflamatorias residentes en la lesión(59), y se considera una molécula proinflamatoria que activa el sistema inmune (60); los niveles de resistina en suero se correlacionan directamente con la obesidad(61). Se reporta una asociación entre inflamación crónica en la lesión de la articulación y los niveles de resistina(62), que se incrementa a nivel local y sistémico posterior al daño articular, y su efecto sobre el cartílago se manifiesta con degradación de la matriz e incentiva la producción de $\operatorname{citosinas}^{(36) .}$ 
La adiponectina, es una proteína de 244 residuos, también llamada GBP28, apM1, Acrp30, o Adipo ${ }^{(63)}$, es sintetizada principalmente por el tejido adiposo en diferentes formas moleculares, los niveles circulantes tienden a ser menores en los pacientes obesos( ${ }^{(64)}$, esta hormona regula el metabolismo energético del organismo, ya que estimula la oxidación de ácidos grasos, reduce los triglicéridos plasmáticos y mejora el metabolismo de la glucosa mediante un aumento de la sensibilidad a la insulina(65), igualmente afecta la gluconeogénesis y la captura celular de glucosa mediante sensibilización a la acción de la insulina(66). Está asociada a múltiples funciones biomoleculares, incluyendo efectos antidiabéticos, anti-inflamatorios y antiarterioescleróticos, esta adipocitoquina a diferencia de la resistina mejora la sensibilidad a la insulina, aumentando el consumo de glucosa e inhibiendo la gluconeogénesis(67); sin embargo, en la OA esta adipocitoquina induce la proliferación de mediadores proinflamatorios como el óxido nítrico, IL-6, IL-8, MMP-3, MMP-9 y la proteína quimioatrayente de monocitos (MCP-1) en los condrocitos ${ }^{(40)}$, por lo anterior los niveles de adiponectina plasmática están más elevados en pacientes con OA en comparación con pacientes sanos, los niveles aumentan a medida que progresa la enfermedad(68,69), no obstante en otros estudios, revelan una relación inversa(70), otro estudio reciente demostró que los niveles de adiponectina en suero no se asociaron con la gravedad de la $\mathrm{OA}$ en manos de poblaciones con las mismas características(71). Esos resultados contradictorios se pueden deber a la fase de evolución y estado de inflamación(72).

La visfatina, es una proteína de aproximadamente 471 aminoácidos y $52 \mathrm{kDa}$, también llamada FEBP (factor de mejora de colonias de células pre-B) y NAMPT (visfatina)(73). Es secretada por los macrófagos y el tejido adiposo visceral(67), en los pacientes obesos, los leucocitos producen mayor cantidad de visfatina, por cuanto existe una relación directa entre obesidad y las concentraciones de visfatina ${ }^{(74)}$. Durante el proceso de inflamación en la $\mathrm{OA}$, los condrocitos producen la visfatina que incrementa la producción de ADAMTS-4, ADAMTS-5, MMP-3, MMP-13 y prostanglandina
E2, las cuales inciden directamente en la degradación del cartílago ${ }^{(75,76)}$. Estudios muestran que los pacientes con OA presentan altas concentraciones de visfatina en el líquido sinovial(77) y se correlaciona con los biomarcadores de degradación tales como el colágeno tipo II y agrecanasa, es decir, que la visfatina estimula actividades catabólicas contribuyendo a la progresión de la enfermedad(78).

\section{Conclusión}

La osteoartrosis se asocia con la sobrecarga sobre las articulaciones, generando procesos inflamatorios, sin embargo, en las articulaciones que no soportan peso también se presenta un proceso inflamatorio, por lo tanto, los estudios se orientan a que la $\mathrm{OA}$, tiene un gran componente metabólico y en su desarrollo participan hormonas y proteínas proinflamatorias. Por tanto, se puede afirmar que existe una amplia relación entre obesidad y $\mathrm{OA}$, debido al incremento exagerado de tejido graso que aumenta el peso sobre las articulaciones activando las vías de señalización celular de la inflamación y en un segundo momento el tejido graso en especial el tejido adiposo blanco, es el principal productor de adipocitoquinas que son unas biomoléculas capaces de potenciar la inflamación; por lo anterior el índice de masa corporal es un predictor de riesgo.

En la OA, las adipocitoquinas potencian la inflamación, contribuyendo a los procesos degenerativos del cartílago y de todas sus estructuras incluyendo al líquido sinovial, donde la sinovitis, la deformidad en la articulación y dolor son signos y síntomas clásicos de la enfermedad. Las adipocitoquinas están relacionadas con la activación de factores inflamatorios tales como interleucina-6 (IL-6), factor de necrosis tumoral alfa $(\mathrm{TNF} \alpha)$, entre otros, cada adipocitoquina realiza esta activación por diferente vía, aunque de algunas se desconoce el mecanismo. Estos desempeñan un rol importante en la etiopatogenia de la OA, sin embargo, se requiere más trabajos de investigación clínica para determinar claramente su papel antes y durante el desarrollo de la 
osteoartrosis, ya que en la mayoría de estudios se hace alusión a que existe una correlación directa entre progresión de la enfermedad y síntesis de adipocitoquinas, debido a que la inflamación y degradación del cartílago y de sus estructuras es mayor en estados avanzados de la misma.

Conflicto de intereses: Los autores declaran no tener ningún conflicto de intereses.

\section{Referencias}

1. Berenbaum F. Osteoarthritis as an inflammatory disease (osteoarthritis is not osteoarthrosis). Osteoarthr Cartilage. 2013;21:16-21.

2. Poole AR, Guilak F, Abramson SB. Etiopathogenesis of Osteoarthritis. En: Moskowitz RW, Altman R, Hochberg M, Buckwalter J, Goldberg VM. (eds) Osteoarthritis: Diagnosis and Medical/Surgical Management. Philadelphia: Lippincott Williams \& Wilkins; 2007.

3. Racine J, Aaron RK. Pathogenesis and epidemiology of osteoarthritis. R I Med J.2013;96(3):19-22.

4. Loeser RF. Age-related changes in the musculoskeletal system and the development of osteoarthritis. Clin Geriatr Med.2010;26:371-386.

5. Bijlsma JW, Berenbaum F, Lafeber FP. Osteoarthritis: an update with relevance for clinical practice. Lancet.2011;377:2115-26.

6. Buckwalter JA, Mankin HJ, Grodzinsky AJ. Articular cartilage and osteoarthritis. Instr Course Lect.2005;54:465-480

7. Lago $\mathrm{R}$, Gomez $\mathrm{R}$, Otero $\mathrm{M}$, et al. A new player in cartilage homeostasis: adiponectin induces nitric oxide synthase type II and pro-inflammatory cytokines in chondrocytes. Osteoarthr Cartilage. 2008;16:1101-9.

8. Lago F, Dieguez C, Gomez-Reino J, et al. The emerging role of adipokines as mediators of inflammation and immune responses. Cytokine Growth Factor Rev. 2007;18: 313-25.

9. Lotz M, Martel-Pelletier J, Christiansen C, Brandi M-L, Bruyère 0 , Chapurlat $R$, et al. Value of biomarkers in osteoarthritis: current status and perspectives. Ann Rheum Dis.2014;90(1061):171-8.

10. Berry PA, Jones SW, Cicuttini FM, Wluka AE, Maciewicz RA. Temporal relationship between serum adipokines, biomarkers of bone and cartilage turnover, and cartilage volume loss in a population with clinical knee osteoarthritis. Arthritis Rheum.2011;63:700-7.

11. Berner HS, Lyngstadaas SP, Spahr A, Monjo M, Thommesen L, Drevon CA, et al. Adiponectin and its receptors are expressed in bone-forming cells. Bone.2004;35:42-9.

12. Hochberg MC. Osteoarthritis: Clinical Features and Treatment. In: Klippel JH. (ed) Primer on the Rheumatic Diseases. Atlanta: Springer; 1997.
13. McNulty A, Miller M, O'Connor S, Guilak F. The Effects of Adipokines on Cartilage and Meniscus Catabolism. Connect Tissue Res.2011;52(6):523-533.

14. Grotle M, Hagen KN, Natvig B, Dahl FA, Kvien TK. Obesity and osteoarthritis in knee, hip and/or hand: an epidemiological study in the general population with 10 years follow-up. BMC Musculoskelet Disord. 2008;9:132.

15. Panel de Expertos de la Sociedad Española de Reumatología (SER). Primer documento de consenso de la Sociedad Española de Reumatología sobre el tratamiento de la artrosis de rodilla. Reumatol Clin. 2005;1:38-48.

16. Leach RE, Baumgard S, Broom J. Obesity: its relationship to osteoarthritis of the knee. Clin Orthop Relat Res. 1973;93:271-273.

17. Raynauld JP, Martel-Pelletier J, Bertjiaume MJ, Beaudoin G, Cjhoqette D, Haraoui B, et al. Long term evaluation of disease progression through the quantitative magnetic resonance imaging of symptomatic knee osteoarthritis patients: correlation with clinical symptoms and radiographic changes. Arthritis Res Ther. 2006;8(1):1-12.

18. Jordan KM, Arden NK, Doherty M, Bannwarth B, Biljsma JWJ, Dieppe P, et al. EULAR recommendations 2003: an evidence based approach to the management of knee osteoarthritis: report of a task force of the standing committee for international clinical studies including therapeutic trials (ESCISIT). Ann Rheum Dis. 2003;62(1):145-55.

19. Houard X, Goldring MB, Berenbaum F. Homeostatic mechanisms in articular cartilage and role of inflammation in osteoarthritis. Current Rheumatology Reports. 2013;15(11):375-385.

20. Guilak F. Biomechanical factors in osteoarthritis. Best Practice and Research: Clinical Rheumatology. 2011;25(6):815-823.

21. Abella V, Scotece M, Conde J, López V, Lazzaro V, Pino J, et al. Adipokines, Metabolic Syndrome and Rheumatic Diseases. J Immunol Res. 2014;20(14):1-14.

22. Griffin TM, Fermor B, Huebner JL, Kraus VB, Rodriguiz RM, Wetsel WC, et al. Diet-induced obesity differentially regulates behavioral, biomechanical, and molecular risk factors for osteoarthritis in mice. Arthritis Res Ther. 2010;12:R130.

23. Flegal KM, Carroll MD, Kit BK, Ogden CL. Prevalence of obesity and trends in the distribution of body mass index among US adults, 1999-2010. JAMA. 2012;307:491-7.

24. Adamczak $M$, Wiecek $A$. The adipose tissue as an endocrine organ. Semin Nephrol. 2013;33(1):2-13.

25. Sirimi N, Goulis DG. Obesity in pregnancy. Hormones (Athens). 2010;9(4):299-306.

26. Otero $M$, Lago $R$, Lago $F$, Casanueva FF, Diéguez $C$, Gómez-Reino JJ, et al. Leptin, from fat to inflammation: old questions and new insights. FEBS Lett. 2005;579:295-301.

27. Sánchez C, Pesesse L, Gabay O, Delcour JP, Msika P, Baudouin $\mathrm{C}$, et al. Regulation of subchondral bone osteoblast metabolism by cyclic compression. Arthritis Rheum. 2012;64(4):1193-1203. 
28. Torzilli PA, Bhargava M, Park S, Chen CT. Mechanical load inhibits IL-1 induced matrix degradation in articular cartilage. Osteoarthr Cartilage. 2010;18:97105.

29. Hopkins SJ, Humphreys M, Jayson MI. Cytokines in synovial fluid. I. The presence of biologically active and immunoreactive IL-1. Clin Exp Immunol. 1988;72(3):422-427.

30. Sanchez C, Deberg MA, Burton S, Devel P, Reginster JY, Henrotin YE. Differential regulation of chondrocyte metabolism by oncostatin $M$ and interleukin-6. Osteoarthr Cartilage. 2004;12(10):801-810.

31. Ding L, Heying E, Nicholson N, Stroud NJ, Homandberg GA, Danping G, et al. Mechanical impact induces cartilage degradation via mitogen activated protein kinases. Osteoarthritis Cartilage. 2010;18(11):15091517.

32. Feldmann M, Brennan FM, Maini RN. Rheumatoid arthritis. Cell. 1996;85:307-310.

33. Tong KM, Chen CP, Huang KC, Shieh DC, Cheng HC, Tzeng CY, et al. Adiponectin increases MMP-3 expression in human chondrocytes through AdipoR1 signaling pathway. J Cell Biochem. 2011;112(5):14311440.

34. Sellam J, Berenbaum F. The role of synovitis in pathophysiology and clinical symptoms of osteoarthritis. Nat Rev Rheumatol. 2010;6:625-635.

35. Goldring M, Otero M. Inflammation in osteoarthritis. Curr Opin Rheumatol. 2011;23(5):471-478.

36. Coggon D, Reading I, Croft P, McLaren M, Barret D, Cooper C. Knee osteoarthritis and obesity. Int J Obes Relat Metab Disord. 2001;25(5):622-627.

37. Bonnet CS, Walsh DA. Osteoarthritis, angiogenesis and inflammation. Rheumatology. 2005;44(1):7-16.

38. Walsh DA, Bonnet CS, Turner EL, Wilson D, Situ M, McWilliams DF. Angiogenesis in the synovium and at the osteochondral junction in osteoarthritis. Osteoarthr Cartilage. 2007;15(7):743-751.

39. Bliddal H, Leeds AR, Stigsgaard L, Astrup A, Christensen R. Weight loss as treatment for knee osteoarthritis symptoms in obese patients: 1- year results from a randomised controlled trial. Ann Rheum Dis. 2011;70(10):1798-1803.

40. Scotece M, Conde J, López V, Lago F, Pino J, GómezReino JJ, et al. Adiponectin and leptin: new targets in infl ammation. Basic Clin Pharmacol Toxicol. 2014;114(1):97-102.

41. Fantuzzi G. Adipose tissue, adipokines, and inflammation. J Allergy Clin Immunol. 2005;115:911920.

42. Zhang Y, Proenca R, Maffei M, Barone M, Leopold L, Friedman JM. Positional cloning of the mouse obese gene and its human homologue. Nature. 1994;372:425432

43. Gualillo O, Eiras S, Lago F, Diéguez C, Casanueva FF. Elevated serumleptin concentrations induced by experimental acute inflammation. Life Sci. 2000;67(20):2433-2441.

44. Roh C, Thoidis G, Farmer S, Kandror K. Identification and characterization of leptin-containing intracellular compartment in rat adipose cells. Am J Physiol Endocrinol Metab. 2000;279:E893-E899.

45. Dumond H, Presle N, Terlain B, Mainard D, Loeuille D, Netter P, Pottie P. Evidence for a key role of leptin in osteoarthritis. Arthritis and Rheumatism. 2003;48(11):3118-3129.

46. Conde J, Scotece M, López V, Abella V, Hermida M, Pino J, et al. Differential expression of adipokines in infrapatellar fat pad (IPFP) and synovium of osteoarthritis patients and healthy individuals. Ann Rheum Dis. 2014;73(3):631-3.

47. Ryan AS, Pratley RE, Elahi D, Goldberg AP. Changes in plasma leptin and insulin action with resistive training in postmenopausal women. Int J Obes. 2000;24:27-32.

48. Sood A, Shore SA. Adiponectin, leptin, and resistin in asthma: basic mechanisms through population studies. J Allergy. 2013:1-15.

49. Ku JH, Lee CK, Joo BS, An BM, Choi SH, Wang TH, et al. Correlation of synovial fluid leptin concentrations with the severity of osteoarthritis. Clin Rheumatol. 2009;28(12):1431-1435.

50. Conde J1, Scotece M, López V, Gómez R, Lago F, Pino J, et al. Adiponectin and leptin induce VCAM-1 expression in human and murine chondrocytes. PLoS One. 2012;7(12):33-5.

51. Gómez R, Scotece M, Conde J, Gómez-Reino JJ, Lago F, Gualillo 0 . Adiponectin and leptin increase IL-8 production in human chondrocytes. Ann Rheumatic Dis. 2011;70(11):2052-2054.

52. Krysiak R, Handzlik-Orlik G, Okopien B. The role of adipokines in connective tissue diseases. Eur J Nutr. 2012;51(5):513-528.

53. Jamaluddin M, Weakley S, Yao Q, Chen C. Resistin: functional roles and therapeutic considerations for cardiovascular disease. B J Pharmacol. 2012;165(3):622-632.

54. Otero M, Lago R, Gómez R, Diéguez C, Lago F, GómezReino J, et al. Towards a pro-inflammatory and immunomodulatory emerging role of leptin. Rheumatology (Oxford). 2006;45:944-50.

55. Trayhurn P, Bing C, Wood IS. Adipose Tissue and Adipokines-Energy Regulation from the Human Perspective. J Nutr. 2006;136:1935S-1939S.

56. Li XC, Tian F, Wang F. Clinical Significance of Resistin Expression in Osteoarthritis: A Meta-Analysis. Biomed Res Int. 2014;2014:208016.

57. Steppan CM, Brown EJ, Wright CM, Bhat S, Banerjee RR, Dai CY, et al. A family of tissue-specific resistin-like molecules. Proc Natl Acad Sci USA. 2001;98(2):502506.

58. Patel L, Buckels AC, Kinghorn IJ, Murdock PR, Holbrook JD, Plumpton C, et al. Resistin is expressed in human macrophages and directly regulated by PPAR $\gamma$ activators. Biochem Biophys Res Commun. 2003;300(2):472-6.

59. Fain J, Cheema P, Bahouth S, Hiler M. Resistin release by human adipose tissue explants in primary culture. Biochem Biophys Res Commun. 2003;300(3):674-678.

60. Barnes KM, Miner JL. Resistin release by human adipose in rodents and humans. Curr Protein Pept Sci. 2009;10:96-107 
61. Degawa-Yamauchi M, Bovenkerk JE, Juliar BE, Watson W, Kerr K, Jones R, et al. Serum resistin (FIZZ3) protein is increased in obese humans. J Clin Endocrinol Metab. 2003;88(11):5452-5455.

62. Senolt L1, Housa D, Vernerová Z, Jirásek T, Svobodová $\mathrm{R}$, Veigl $\mathrm{D}$, et al. Resistin in rheumatoid arthritis synovial tissue, synovial fluid and serum. Ann Rheum Dis. 2007;66(4):458-463.

63. Garaulet M, Hernandez-Morante JJ, de Heredia FP, Tebar FJ. Adiponectin, the controversial hormone. Public Health Nutr. 2007;10:1145-1150.

64. Oh DK, Ciaraldi T, Henry R. Adiponectin in health and disease. Diabetes Obes Metab. 2007;9(3):282-289.

65. Palomera X, Pérez A, Blanco-Vaca F. Adiponectina: un nuevo nexo entre obesidad, resistencia a la insulina y enfermedad cardiovascular. Med Clin (Barc). 2005;124(10):388-395.

66. Hernández-Valencia $\mathrm{M}$, Zárate $\mathrm{A}$, Galván RE. Concentración de la adiponectina en perimenopáusicas. Ginecol Obstet Mex. 2008;76(8):450-453.

67. Ranheim T, Haugen F, Staff AC, Braekke K, Harsem NK, Drevon CA. Adiponectin is reduced in gestational diabetes mellitus in normal weight women. Acta Obstet Gynecol Scand. 2004;83(4):341-347.

68. Lauberg TB, Frystyk J, Ellingsen T, Hansen IT, Jorgensen A, Tarp U, et al. Plasma adiponectin in patients with active, early, and chronic rheumatoid arthritis who are steroid-and disease-modifying antirheumatic drug-naive compared with patients with osteoarthritis and controls. J Rheumatol. 2009;36(9):1885-1891.

69. Filková M, Lisková M, Hulejová H, Haluzík M, Gatterová J, Pavelková A, et al. Increased serum adiponectin levels in female patients with erosive compared with nonerosive osteoarthritis. Ann Rheum Dis. 2009;68(2):295-296.

70. Honsawek S, Chayanupatkul M. Correlation of plasma and synovial fluid adiponectin with knee osteoarthritis severity. Arch Med Res. 2010;41(8):593-598.

71. Chung CP1, Long AG, Solus JF, Rho YH, Oeser A, Raggi P, et al. Adipocytokines in systemic lupus erythematosus: relationship to inflammation, insulin resistance and coronary aterosclerosis. Lupus. 2009;18(9):799-806.

72. Massengale M, Lu B, Pan JJ, Katz JN, Solomon DH. Adipokine hormones and hand osteoarthritis: radiographic severity and pain. PLoS One. 2012;7(10):e47860.

73. Katsiougiannis S, Tenta R, Skopouli FN. Activation of AMP-activated protein kinase by adiponectin rescues salivary gland epithelial cells from spontaneous and interferon- $\gamma$ - induced apoptosis. Arthritis Rheum. 2010;62(2):414-419.

74. Gómez R, Conde J, Scotece M, Gómez-Reino JJ, Lago F, Gualillo O. What's new in our understanding of the role of adipokines in rheumatic diseases?. Nat Rev Rheumatol. 2011;7:528-536.

75. Brentano F, Schorr O, Ospelt C, Stanczyk J, Gay RE, Gay $\mathrm{S}$, Kyburz D. Pre-B cell colony-enhancing factor/visfatin, a new marker of inflammation in rheumatoid arthritis with proinflammatory and matrix degrading activities. Arthritis Rheum. 2007;56:28292839.

76. Little CB, Hunter DJ. Post-traumatic osteoarthritis: from mouse models to clinical trials. Nat Rev Rheumatol. 2013;9:485-97.

77. Meier FM1, Frommer KW, Peters MA, Brentano F, Lefèvre $\mathrm{S}$, Schröder D, et al. Visfatin/pre-B-cell colonyenhancing factor (PBEF), a proinflammatory and cell motility-changing factor in rheumatoid arthritis. J Biol Chem. 2012;287:28378-28385.

78. Yang S, Ryu JH, Oh H, Jeon J, Kwak JS, Kim JH, et al. NAMPT (visfatin), a direct target of hypoxia inducible factor- $2 \alpha$, is an essential catabolic regulator of osteoarthritis. Ann Rheum Dis. 2015;74(3):595-602. 